\title{
Intravenous magnesium sulfate for acute wheezing in young children: a randomised double-blind trial
}

\author{
Hannele Pruikkonen ${ }^{1}$, Terhi Tapiainen ${ }^{1,2}$, Merja Kallio ${ }^{1,2}$, Teija Dunder ${ }^{1,2}$, \\ Tytti Pokka ${ }^{1,2}$, Matti Uhari ${ }^{1,2}$ and Marjo Renko ${ }^{1,2}$ \\ Affiliations: ${ }^{1}$ PEDEGO Research Unit and Medical Research Center Oulu, University of Oulu, Oulu, Finland. \\ ${ }^{2}$ Dept of Children and Adolescents, Oulu University Hospital, Oulu, Finland. \\ Correspondence: Hannele Pruikkonen, PEDEGO Research Unit and Medical Research Center Oulu, University \\ of Oulu, BOX 5000, FIN-90014, Oulu, Finland. E-mail:hannele.pruikkonendoulu.fi
}

@ERSpublications

Intravenous magnesium sulfate was ineffective in treating acute severe virus-induced wheezing in young children http://ow.ly/sfcF30hHc0e

Cite this article as: Pruikkonen $\mathrm{H}$, Tapiainen T, Kallio $\mathrm{M}$, et al. Intravenous magnesium sulfate for acute wheezing in young children: a randomised double-blind trial. Eur Respir J 2018; 51: 1701579 [https://doi. org/10.1183/13993003.01579-2017].

ABSTRACT Magnesium sulfate has been shown to be an effective treatment in older children with asthma exacerbations, but it has not been investigated in acute severe virus-induced wheezing in young children.

The study enrolled 61 children aged 6 months to 4 years. Inclusion criteria were severe wheezing, classified as a score of $\geqslant 6$ points as assessed by the Respiratory Distress Assessment Instrument (RDAI) after initial treatment with salbutamol, and the symptoms of acute viral infection. The children were randomly allocated to receive either an infusion of magnesium sulfate $\left(40 \mathrm{mg} \cdot \mathrm{kg}^{-1}\right)$ or $0.9 \%$ sodium chloride as a placebo infusion for $20 \mathrm{~min}$. Primary outcome measure was mean change in RDAI scores from baseline to $6 \mathrm{~h}$ after the treatment.

Change in the severity of wheezing from baseline to $6 \mathrm{~h}$ after the treatment, as measured by mean $\pm \mathrm{SD}$ RDAI scores, was $4.7 \pm 2.6$ in the magnesium sulfate group and $4.2 \pm 4.2$ in the placebo group (difference $0.5,95 \% \mathrm{CI}-1.3$ to $2.3, \mathrm{p}=0.594$ ).

Intravenous magnesium sulfate was ineffective in treating acute severe virus-induced wheezing in young children, in contrast to the previous efficacy demonstrated in older children.

Received: Aug 032017 | Accepted after revision: Nov 192017

This study was registered at Clinicaltrials.gov with identifier number NCT01383655.

Support statement: Hannele Pruikkonen received a grant from the Alma and K.A. Snellman Foundation and a grant from the Finnish Medical Association (Duodecim Northern Ostrobothnia) to support the reporting phase of this study. Funding information for this article has been deposited with the Crossref Funder Registry.

Conflict of interest: Disclosures can be found alongside this article at erj.ersjournals.com

Copyright @ERS 2018 


\section{Introduction}

Acute wheezing during viral respiratory infections is one of the most common causes of emergency room visits and hospital admissions among infants and preschool children [1]. Bronchodilators and systemic corticosteroids are not as effective in young children as they are in older children with severe exacerbations of asthma [2,3]. The difference in the efficacy of medications according to patient age suggests that virus-induced wheezing and asthma have different pathophysiologies.

Intravenous magnesium sulfate has been successfully used in older children with life-threatening asthma exacerbations and in those whose symptoms remain severe after $1 \mathrm{~h}$ of intensive conventional therapy [4-11]. Magnesium causes smooth muscle relaxation by blocking the release of calcium [12]. Magnesium also acts through other mechanisms, including stabilisation of T-cells, inhibition of mast cell degranulation, inhibition of acetylcholine release inhibition, and stimulation of nitric oxide and prostacyclin synthesis, thereby reducing airflow obstruction [12].

Young children with acute virus-induced severe wheezing pose a significant therapeutic challenge in emergency care settings, and there is a need for effective treatment. Magnesium sulfate has been shown to be an effective treatment in older children with acute asthma exacerbations. Thus, we designed a randomised, double-blind, placebo-controlled study to evaluate the efficacy of an intravenous infusion of magnesium sulfate as an additional treatment in children aged 6 months to 4 years with severe acute wheezing during respiratory viral infections. Children with inspiratory crackles and those with chronic asthma symptoms between viral infection episodes were excluded because we focused on virus-induced expiratory wheezing.

\section{Materials and methods}

Study design and study subjects

This was a randomised (1:1) double-blind placebo-controlled, parallel-group trial in children aged 6 months to 4 years with wheezing during viral respiratory infections conducted in Oulu University Hospital, Finland (figure 1). The patients were recruited from March 2011 to December 2015 at the Department of Children and Adolescents, Oulu University Hospital, Oulu, Finland. Oulu University

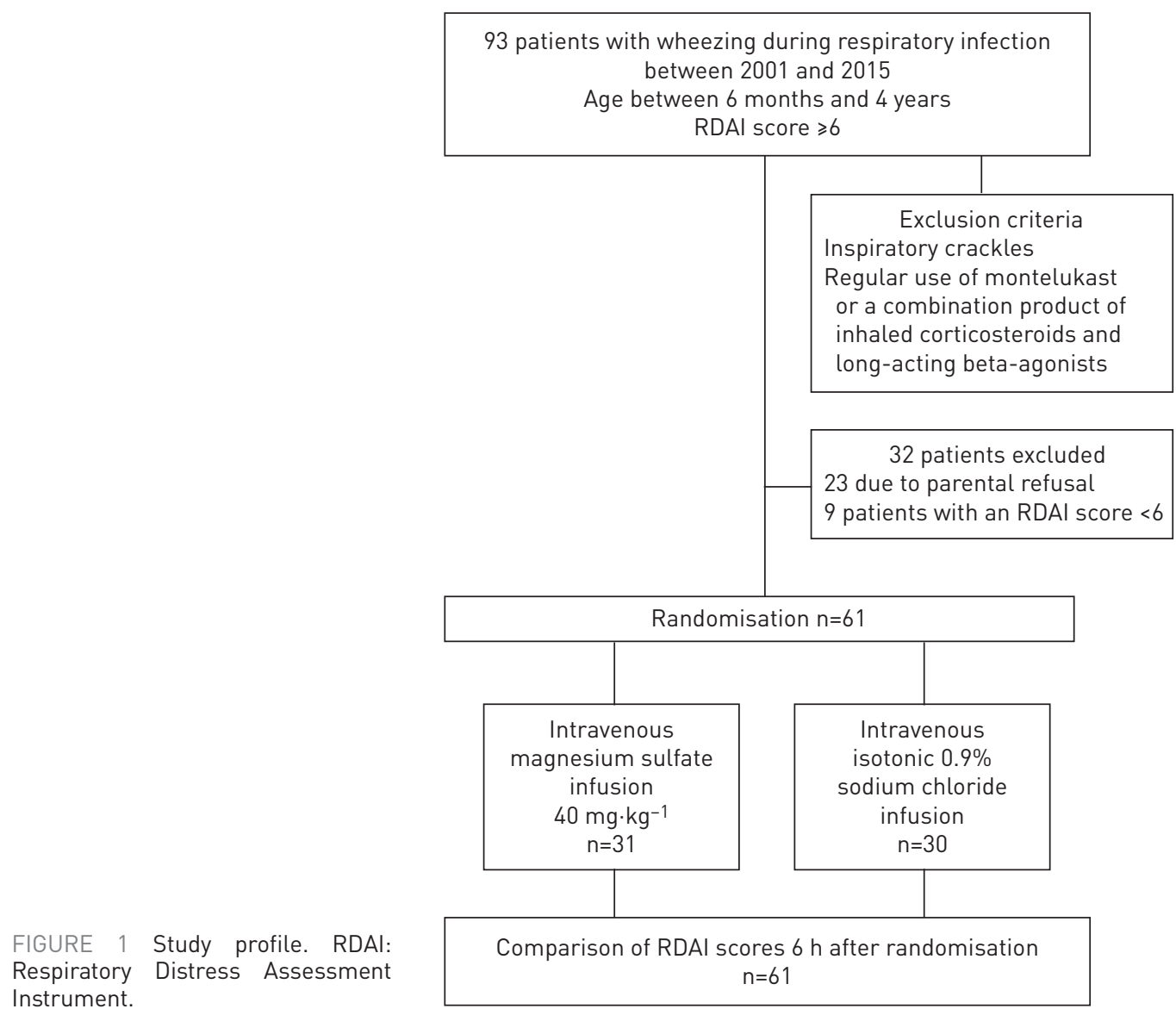


Hospital is as a tertiary paediatric hospital, which serves a population of 164426 individuals aged $<18$ years, and is the only paediatric hospital serving a population of 82753 children $<15$ years. The trial was registered at ClinicalTrials.gov (NCT01383655). The ethics committee of the Northern Ostrobothnia Hospital District, Finland approved the study protocol. Only children whose parents provided written informed consent participated in the trial.

Eligible participants were children aged 6 months to 4 years with wheezing during viral respiratory infections. Inclusion criteria were acute severe wheezing, i.e. respiratory distress classified as a score of $\geqslant 6$ points on the Respiratory Distress Assessment Instrument (RDAI), and the symptoms of acute viral respiratory tract infection (wheezing, cough, rhinitis and fever) (table 1) [13]. The RDAI score was chosen for this study because it was used in the earlier study evaluating children younger than 24 months with wheezing [14]. All the patients were initially treated with a $2.4 \mathrm{mg}$ dose of salbutamol using a pressurised metered-dose spacer chamber (Babyhaler; GlaxoSmithKline, Stockley Park, UK) with a mask. Salbutamol was administered at a dose of $0.6 \mathrm{mg}$ every $20 \mathrm{~min}$ for the first hour (total $2.4 \mathrm{mg}$ ). Each $0.6 \mathrm{mg}$ dose was administered in three $0.2 \mathrm{mg}$ aliquots in the spacer, and the mask was kept in place until the child had taken five breaths [15]. Only children who suffered acute severe wheezing symptoms (respiratory distress) after initial treatment with salbutamol were eligible for the trial. The personnel in the paediatric emergency clinic were instructed to immediately contact one of the three physicians involved in the study if an eligible patient was admitted to the hospital. These physicians were responsible for recruiting the patients and informing the parents and caregivers about the study, checking the inclusion and exclusion criteria, evaluating the patients' RDAI scores, and obtaining written informed consent. We used a multiple PCR assay (Seeplex RV12; Seegene, Seoul, Korea) to analyse nasopharyngeal samples to determine the cause of viral respiratory infection from all patients attending the study.

Exclusion criteria were presence of chronic severe asthma symptoms between viral infection episodes; gestational age <35 weeks; current or previous diagnosis of bronchopulmonary dysplasia, immunodeficiency, congenital heart defects, neurological disease, laryngitis or foreign body of the respiratory tract; RDAI score $<6$ points; and presence of probable pneumonia based on inspiratory crackles on auscultation. At the beginning of the study, patients with regular usage of inhaled corticosteroids were excluded to avoid recruiting patients with chronic regular asthma symptoms between viral infection episodes. However, the study protocol was revised after 6 months of recruitment, and these patients were included in the trial because inhaled corticosteroids are commonly used for the prevention of recurrent viral infection-induced wheezing episodes. Altogether, 11 patients were recruited before the revision of the study protocol. As the aim of this study was to identify an additional treatment for severe wheezing induced by recurrent viral infections, patients with severe chronic asthma symptoms requiring regular usage of montelukast or a combination of inhaled corticosteroids and long-acting beta-agonists were excluded.

After obtaining informed consent, an intravenous access tube was inserted for the study treatment, unless already inserted by the emergency room physician. The physicians recorded the heights and weights of all the participants at study entry to ensure that participants had normal nutritional status. All other medical

\begin{tabular}{|c|c|c|c|c|c|c|}
\hline & \multicolumn{5}{|c|}{ Points } & \multirow{2}{*}{$\begin{array}{c}\text { Maximum } \\
\text { points }\end{array}$} \\
\hline & 0 & 1 & 2 & 3 & 4 & \\
\hline \multicolumn{7}{|l|}{ Wheezing } \\
\hline Expiration & None & End & $1 / 2$ & $3 / 4$ & All & 4 \\
\hline Inspiration & None & Part & All & & & 2 \\
\hline Location & None & $\begin{array}{c}\text { Segmental: } \leqslant 2 \text { of } 4 \\
\text { lung fields }\end{array}$ & $\begin{array}{l}\text { Diffuse: } \geqslant 3 \text { of } 4 \\
\text { lung fields }\end{array}$ & & & 2 \\
\hline \multicolumn{7}{|l|}{ Retractions } \\
\hline Supraclavicular & None & Mild & Moderate & Marked & & 3 \\
\hline Intercostal & None & Mild & Moderate & Marked & & 3 \\
\hline Subcostal & None & Mild & Moderate & Marked & & 3 \\
\hline
\end{tabular}

The maximum total number of points for wheezing was 8 , and the maximum total number of points for retractions was 9 . All the children with silent expiratory sounds during auscultation were assigned the maximum score for wheezing. 
treatments, including inhalations of salbutamol, systemic corticosteroids and intensive care, were decided by the clinical paediatrician and not by the study physicians. Supplemental oxygen was provided for patients with an oxygen saturation of $\leqslant 92 \%$ as determined by pulse oximetry.

\section{Study products, blinding, randomisation and data monitoring}

The patients were randomly allocated to receive either an intravenous infusion of magnesium sulfate $\left(40 \mathrm{mg} \cdot \mathrm{kg}^{-1}\right.$ ) or a placebo infusion of $0.9 \%$ sodium chloride for $20 \mathrm{~min}$. The maximum magnesium sulfate dose was $2 \mathrm{~g}$. At the time this study was planned, five studies evaluating the efficacy of intravenous magnesium sulfate in the the treatment of acute paediatric asthma had been published. Those studies were randomised double-blind trials, and the dose of intravenous magnesium ranged from $25 \mathrm{mg} \cdot \mathrm{kg}^{-1}$ to $100 \mathrm{mg} \cdot \mathrm{kg}^{-1}$ [4-8]. We chose the dose of $40 \mathrm{mg} \cdot \mathrm{kg}^{-1}$ based on these studies. Randomisation was performed in blocks of four by a biostatistician, using the StatsDirect v2 ${ }^{\circ}$ (StatsDirect Ltd, Cheshire, UK) statistical program [16]. Block randomisation was used to ensure that there were similar numbers of patients in the magnesium sulfate and placebo groups during different epidemic seasons.

The products used in the study were prepared in Oulu University Hospital pharmacy, using $100 \mathrm{~mL}$ Ecoflac (B. Braun, Boulogne, France) bottles containing $0.9 \%$ sodium chloride. The pharmacists in the hospital used sterile instruments to open the bottles, and removed $10 \mathrm{~mL}$ of the volume. For the active bottles, magnesium sulfate $8 \mathrm{~mL}$ (concentration of $246 \mathrm{mg} \cdot \mathrm{mL}^{-1}$ ), Addex-Magnesium (Fresenius Kabi, Uppsala, Sweden), was then added, resulting in a magnesium sulfate solution with a concentration of $20 \mathrm{mg} \cdot \mathrm{mL}^{-1}$. For the placebo bottles, $0.9 \%$ sodium chloride $(8 \mathrm{~mL})$ was added. The magnesium and placebo products were packed in identical bottles. In both cases, the fluid was transparent and water-like. The bottles were coded with the study number according to a randomised sequence, which was kept in the pharmacy of the hospital. The pharmacy staff delivered the coded bottles in numerical order. The study doctors, physicians in charge, nurses and caregivers were blinded to the study medication until the data entry was complete and the data were ready for the final analysis.

During the intravenous 20-min infusion, the study physicians closely monitored the patients to record possible side effects, such as nausea, somnolence, tachycardia, facial redness, epigastric redness and pain, and burning or redness at the site of the cannula, all of which have been associated with intravenous magnesium infusions [17]. The study physicians observed and recorded RDAI scores, heart rates, respiratory rates and oxygen saturation levels, as well as patient condition (alert and calm/crying/sleeping) and the presence of silent respiratory sounds during auscultation. The data were recorded at baseline, $20 \mathrm{~min}, 1 \mathrm{~h}, 2 \mathrm{~h}$ and $6 \mathrm{~h}$ after the treatment. After the first $6 \mathrm{~h}$, the data recording continued at $12 \mathrm{~h}$ intervals until the patient was discharged.

\section{Primary outcome}

The primary outcome was the change in the mean RDAI score from baseline to $6 \mathrm{~h}$ after the study treatment. We reported separately the results of the mean RDAI scores from patients who tested positive for rhinovirus, as wheezing in early childhood caused by rhinovirus has been found to be a strong risk factor for later diagnosis of asthma [18].

\section{Secondary outcomes}

Secondary outcomes were the proportion of children needing supplemental oxygen, proportion of children requiring treatment in the intensive care unit, duration of hospitalisation, and occurrence of revisits and re-admissions within 30 days after the study treatment.

\section{Sample size}

The sample size calculation was based on our earlier experience of 60 children, with a mean age of 1 year, who experienced wheezing during viral respiratory infections in our emergency room. We regarded the difference between groups as clinically significant if the mean RDAI score at $6 \mathrm{~h}$ after the study treatment was 4.0 for the children receiving magnesium sulfate and $5.4 \pm 1.7$ for the children receiving placebo treatment. We calculated that 26 children were required for both groups to identify this difference with a type I error of 0.05 and a type II error of 0.20 (power of $80 \%$ ).

\section{Statistical analyses}

Continuous variables were presented as mean and standard deviation and categorical variables were presented as count and proportion. All the analyses were based on the intention-to-treat principle. $t$-test was used to determine the statistical significance of between-group differences in the continuous variables. The standard normal deviate test was used to compare proportions between the study groups. We calculated 95\% confidence intervals for the differences. Data on the time from baseline to revisits and readmissions are presented as Kaplan-Meier survival curves, and the difference between the groups was 
tested by the log rank test. A logistic regression analysis, which was adjusted for the RDAI score at study entry, was used to evaluate the impact of the magnesium sulfate treatment on the need for supplemental oxygen, intensive care, revisits and re-admissions. Two-sided p-values are reported throughout. The analyses were carried out with IBM SPSS Statistics for Windows, Version 22.0 (IBM Corp., Armonk, NY).

\section{Results \\ Study population}

In total, 93 patients met the inclusion criteria and were invited to participate in this trial between March 2011 and December 2015. Of these, 23 families refused to participate, and nine patients had an RDAI score of $<6$ points, thus, the final study consisted of 61 children who were randomly allocated to receive either magnesium sulfate $(n=31)$ or placebo $(n=30)$ (figure 1). The most common reasons for parental refusal to participate in the study were the need to insert an intravenous cannula $(n=9)$ and the fear of side effects of the medication $(n=6)$. RDAI scores for the analysis of the primary outcome were available for all randomised participants. The groups were well balanced in terms of age, sex and patient history (table 2). In total, $44 \%$ of the parents reported smoking (table 2). The mean \pm sD age of the participants was $1.8 \pm 0.9$ years and $53 \%$ were boys. All the participants had an acute respiratory infection at study entry, while $90 \%$ had cough, $80 \%$ had rhinitis and $54 \%$ had fever. Rhinovirus was identified in 44 of 61 children (72\%) and any respiratory virus in 54 of 61 children (89\%) (table 2). The majority of the patients had severe wheezing, with a mean RDAI score at baseline of $11.1 \pm 2.7$. The patients randomised to the magnesium sulfate group had lower RDAI scores at study entry (tables 2 and 3).

\section{Primary outcome}

The mean change in the RDAI scores from baseline to $6 \mathrm{~h}$ was not significantly different between the magnesium sulfate and placebo groups (figure 2), being $4.7 \pm 2.6$ in the magnesium sulfate group and $4.2 \pm 4.2$ in the placebo group (difference $0.5,95 \%$ CI -1.3 to $2.3 ; \mathrm{p}=0.594$ ) (table 3 ).

TABLE 2 Baseline characteristics of the patient groups

\begin{tabular}{lcc} 
& Magnesium (n=31) & Placebo (n=30) \\
\hline Age years & $1.7 \pm 0.8$ & $2.0 \pm 1.0$ \\
6-12 months & $6(19)$ & $5(17)$ \\
$1-2$ years & $16(52)$ & $9(30)$ \\
$2-3$ years & $7(23)$ & $11(37)$ \\
$3-4$ years & $2(7)$ & $5(17)$ \\
Male sex & $17(55)$ & $15(50)$ \\
Birth weight g & $3346 \pm 651$ & $3544 \pm 469$ \\
Duration of respiratory infection days & $3.1 \pm 3.7$ & $3.0 \pm 3.6$ \\
Duration of wheezing days & $1.0 \pm 0.7$ & $1.1 \pm 1.4$ \\
Number of earlier episodes of wheezing & $5.3 \pm 6.7$ & $6.1 \pm 6.0$ \\
Regular use of an inhaled corticosteroid & $7(23)$ & $6(20)$ \\
Patient history of doctor diagnosed atopy & $11(36)$ & $6(20)$ \\
Patient history of doctor diagnosed food allergy & $3(10)$ & $6(20)$ \\
Parental smoking & $14(45)$ & $13(43)$ \\
Mother with a diagnosis of asthma & $6(19)$ & $4(13)$ \\
Father with a diagnosis of asthma & $2(7)$ & $5(17)$ \\
Pets at home & $13(42)$ & $13(43)$ \\
Number of doses of short-acting beta-agonists & $6.2 \pm 3.7$ & $6.2 \pm 4.5$ \\
before the study treatment & & $12(40)$ \\
Supplemental oxygen before the study treatment & $12(39)$ & $15(50)$ \\
Viral aetiology of wheezing & & $3(10)$ \\
Rhinovirus & $22(71)$ & $3(10)$ \\
Rhinovirus and other virus & $4(13)$ & $2(7)$ \\
Respiratory syncytial virus & $2(7)$ & $0(0)$ \\
Adenovirus & $0(0)$ & $2(7)$ \\
Coronavirus Oc43 & $1(3)$ & $5(17)$ \\
Bocavirus & $0(0)$ & $2(7)$ \\
Negative & & \\
\hline
\end{tabular}

Data are presented as mean \pm sD or $\mathrm{n}(\%)$. 
TABLE 3 Outcomes of the patients in the magnesium sulfate and placebo groups

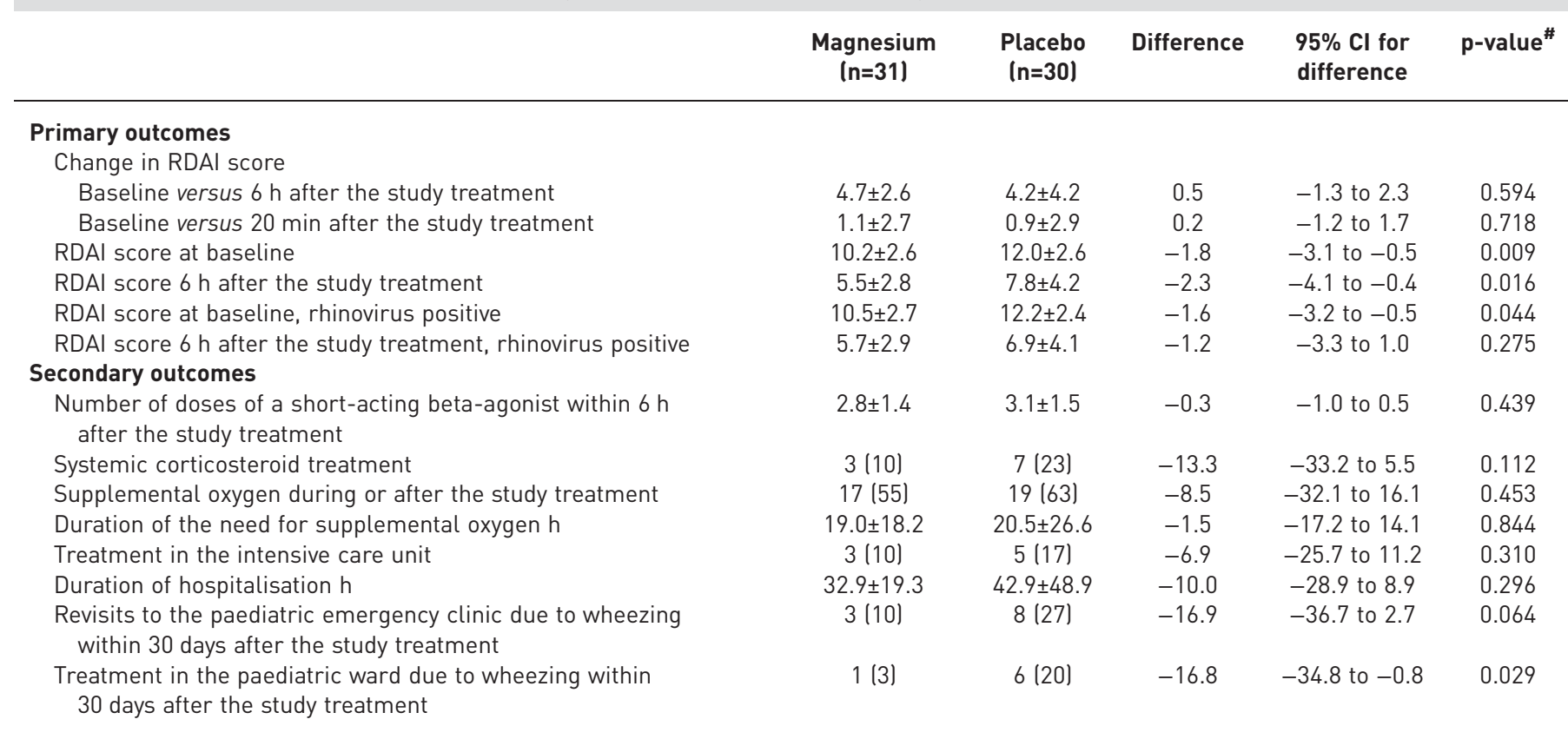

Data are presented as mean \pm SD or $\mathrm{n}(\%)$, unless otherwise stated. ${ }^{\#}$ : standard normal deviate test or t-test, where appropriate. RDAl: Respiratory Distress Assessment Instrument.

\section{Secondary outcomes}

There were no between-group differences in the number of patients requiring supplemental oxygen or treatment in the intensive care unit (table 3). The duration of hospitalisation did not differ significantly between the two groups, being $32.9 \mathrm{~h} \pm 19.3 \mathrm{in}$ the magnesium sulfate group and $42.9 \pm 48.9 \mathrm{~h}$ in the placebo group (difference $-10.0,95 \%$ CI -28.9 to $8.9 ; \mathrm{p}=0.296$ ) (table 3). One patient in the placebo group required mechanical ventilation during hospitalisation.

Within the 30 days after discharge, there were fewer revisits $(n=3 ; 10 \%)$ for the magnesium sulfate group than for the placebo group ( $\mathrm{n}=8 ; 27 \%$ ) (difference $-17 \%, 95 \% \mathrm{CI}-36.7$ to $2.7 ; \mathrm{p}=0.064$ ). There were also fewer readmissions $(n=113 \%)$ for the magnesium sulfate group than for the placebo group $(n=6 ; 20 \%)$ within the 30 days after discharge, (difference $-17 \%, 95 \% \mathrm{CI}-34.8$ to $-0.8 ; \mathrm{p}=0.029$ ) (table 3 ). However, these findings were not significant when the logistic regression analysis was adjusted for RDAI scores at study entry. In the logistic regression analysis, intravenous magnesium sulfate did not affect the need for supplemental oxygen or intensive care.

One patient in the magnesium sulfate group had facial redness and redness around the site of the intravenous cannula during the first $15 \mathrm{~min}$ of the intravenous infusion. No other adverse events were recorded in the children in either group. Heart rate, respiratory rate and oxygen saturation measurement did not differ between the groups at baseline or at $20 \mathrm{~min}$ after receiving the study treatment (table 4).

\section{Discussion}

Intravenous magnesium sulfate was not an effective additional therapy in treating severe virus-induced wheezing in this double-blind randomised controlled trial of children aged of 6 months to 4 years.

Earlier studies and meta-analyses demonstrated the efficacy of magnesium sulfate in older children with severe asthma exacerbations [9, 19-21]. Previous meta-analyses concluded that the use of intravenous magnesium sulfate as an adjunct therapy in children with acute severe asthma significantly reduced the number of hospital admissions, with a reported risk ratio for hospitalisation of $0.32-0.70[9,19,21]$. In earlier studies, children with asthma also had a wide age range, from 1 year to 18 years and from 6 years to 18 years $[4-8,10,11]$.

The main reason for the lack of efficacy of magnesium sulfate in the present study may be that the pathophysiology of asthma is different from the pathophysiology of viral wheeze in early childhood. Biopsy studies of children with asthma have reported the presence of thickening of the epithelial reticular 

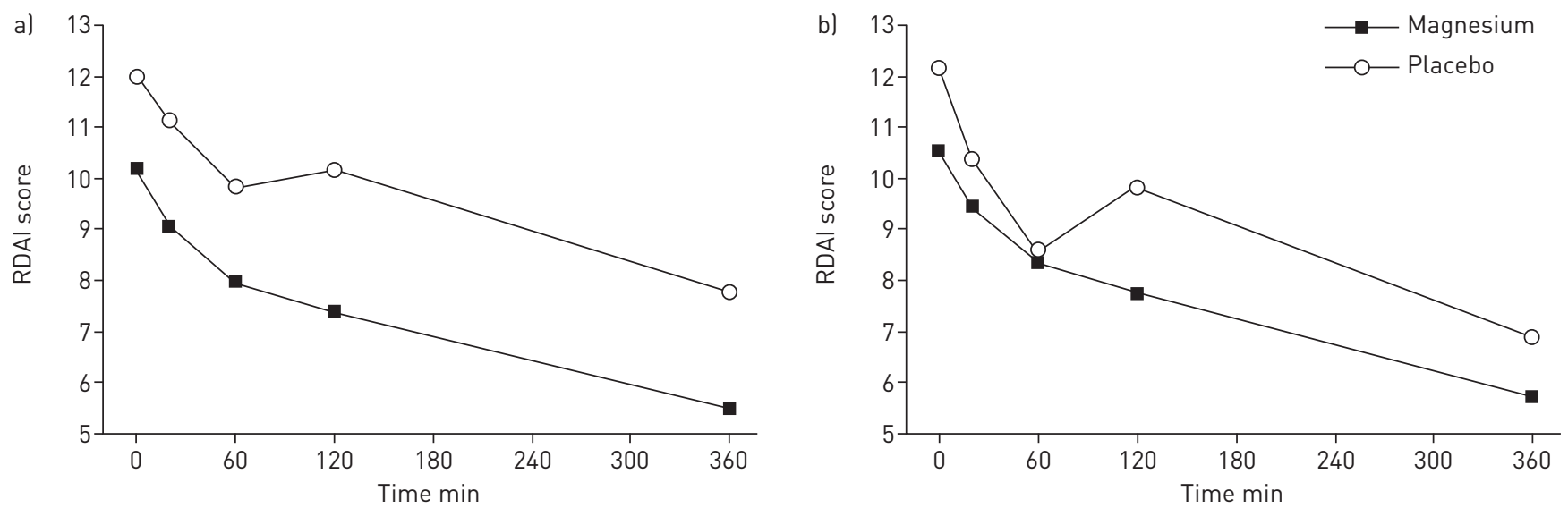

FIGURE 2 Respiratory Distress Assessment Instrument (RDAI) scores for magnesium sulfate and placebo groups over time (a). The RDAl scores of both the magnesium sulfate group and placebo group showed a significant difference at baseline and $6 \mathrm{~h}$ after the study treatment. The changes in RDAl scores did not differ over time between the groups. RDAl scores for magnesium sulfate and placebo groups with rhinovirus infection over time (b).

basement membrane and eosinophilic inflammation in those aged 6 years to 16 years, whereas these characteristics are absent in symptomatic infants younger than 2 years with reversible airway obstruction [22]. The term "wheeze" is a clinical symptom describing a continuous high-pitched sound emitted from the chest during expiration, which is the result of narrowing the intrathoracic airways and expiratory flow limitation [23]. The validity of the different scores in evaluating the respiratory distress in young children is limited, and a recent study concluded that respiratory rate, oxygen need and presence of retractions are more useful tools for predicting the need for hospital admission in young children [24]. The clinical entity of preschool wheeze classically affects children younger than 5 years. Children with wheeze and/or crackles during viral infection who are under the age of 12 months in Europe, or under the age of 24 months in the USA, are often diagnosed with bronchiolitis [25]. Thus, in the present study, some of the patients may have fulfilled the criteria for a diagnosis of bronchiolitis, as the diagnostic criteria for bronchiolitis and virus-induced wheezing in young children are overlapping. However, we excluded patients with inspiratory crackles and included only young children with acute severe virus-induced expiratory wheezing, which was probably caused by reversible bronchial obstruction. These exclusion criteria would have made smooth muscle relaxation a plausible mechanism for the efficacy of magnesium sulfate in virus-induced wheezing in young children. A randomised controlled trial of 162 infants with a median age of 3.7 months who had bronchiolitis found that patients who received intravenous magnesium sulfate had similar mean bronchiolitis severity scores over time compared with the control group [26]. The

TABLE 4 Patient monitoring

\begin{tabular}{|c|c|c|c|c|c|}
\hline & $\begin{array}{l}\text { Magnesium } \\
{[(n=31]}\end{array}$ & $\begin{array}{l}\text { Placebo } \\
(n=30)\end{array}$ & Difference & $\begin{array}{l}95 \% \mathrm{Cl} \text { for } \\
\text { difference }\end{array}$ & p-value ${ }^{\#}$ \\
\hline \multicolumn{6}{|l|}{ Patient condition at baseline } \\
\hline Sleeping & $11(36)$ & $2(7)$ & 29.4 & 8.9 to 47.9 & 0.006 \\
\hline Crying & $2(7)$ & $10(33)$ & -26.8 & -46.2 to -7.4 & 0.006 \\
\hline \multicolumn{6}{|l|}{ Patient condition 20 min after the study treatment } \\
\hline Crying & 9 (29) & $7(23)$ & 5.7 & -16.8 to 27.6 & 0.578 \\
\hline Heart rate at baseline & $144.7 \pm 13.8$ & $150.3 \pm 14.8$ & -5.6 & -13.0 to 1.7 & 0.129 \\
\hline Heart rate 20 min after the study treatment & $145.0 \pm 15.7$ & $145.2 \pm 14.5$ & -0.2 & -7.9 to 7.5 & 0.959 \\
\hline Respiratory rate at baseline & $50.1 \pm 11.2$ & $51.4 \pm 8.9$ & -1.3 & -6.5 to 3.9 & 0.608 \\
\hline Respiratory rate $20 \mathrm{~min}$ after the study treatment & $48.2 \pm 11.5$ & $48.6 \pm 9.8$ & -0.4 & -5.9 to 5.1 & 0.883 \\
\hline Oxygen saturation at baseline $\%$ & $92.9 \pm 3.0$ & $93.1 \pm 3.1$ & -0.2 & -1.8 to 1.3 & 0.768 \\
\hline
\end{tabular}

Data are presented as mean \pm SD or $\mathrm{n}(\%)$, unless otherwise stated. ${ }^{\#}$ : standard normal deviate test or t-test, where appropriate. 
same study found that the infants who received magnesium sulfate were more often readmitted in the 2 weeks post treatment [26].

Nearly $30 \%$ of all children experience a wheezing episode by the age of 3 years, and $30 \%$ of preschoolers with recurrent wheeze are diagnosed with asthma by the age of 6 years [27, 28]. There are overlapping areas between infants with bronchiolitis, children with recurrent viral wheezing episodes and patients with a clear asthma presentation. We excluded patients with severe chronic asthma symptoms between viral infection episodes, as well as all infants with inspiratory crackles. In the present study, among the patients who received intravenous magnesium sulfate, there were significantly fewer readmissions due to wheezing in the 30 days post treatment, pointing to a possible long-lasting effect of magnesium sulfate. This finding has not been reported in earlier studies. However, this finding was not statistically significant when the analysis was adjusted for RDAI scores at study entry.

Exposure to cigarette smoke is a major risk factor for virus-induced acute respiratory tract infections in children younger than 3 years and for recurrent wheezing at the age of 3 years [29]. In the present study, this risk factor was also present in a relative large number of cases, with $44 \%$ of parents reporting smoking. Currently, up to $16 \%$ of working-aged Finns smoke daily [30]. Exposure to cigarette smoke blunts the response of airway epithelial cells to rhinovirus infection, leading to increased viral replication and production, potentially by interfering with the interferon pathway and the transcriptional activation of antiviral genes [31].

The strength of the present study is its randomised double-blind and placebo-controlled design. An additional strength is that we were able to collect data on outcome measurements from all the participants randomised to the study treatment. We successfully reached the sample size calculated before the study, but a larger sample size might have detected smaller differences between the groups. To take account of the sample size, the differences between the groups were expressed with 95\% CIs. In addition, the patients randomised to the magnesium sulfate group had more rhinovirus infections but less severe wheezing episodes compared with the placebo group. The intravenous magnesium dose was $40 \mathrm{mg} \cdot \mathrm{kg}^{-1}$ in this study. After we had completed our study, a retrospective pharmacokinetic study was published suggesting that a dose of 50-75 mg. $\mathrm{kg}^{-1}$ intravenous magnesium should be administered to achieve a hypothesised target therapeutic range [32].

In the present study, intravenous magnesium sulfate administered in the early course of the infection to children aged 6 months to 4 years with severe virus-induced wheezing episodes conferred no additional benefit in terms of wheezing severity, need for supplemental oxygen, need for intensive care or duration of hospitalisation. Therefore, intravenous magnesium sulfate should not be administered to treat virus-induced wheezing among preschool children. The pathophysiology of virus-induced wheeze in infants and preschool children is likely to be different from the pathophysiology of asthma in older children. This would explain the difference in the efficacy of intravenous magnesium sulfate in different age groups with wheezing-related diseases.

\section{References}

1 Kuehni CE, Davis A, Brooke AM, et al. Are all wheezing disorders in very young (preschool) children increasing in prevalence? Lancet 2001; 357: 1821-1825.

2 Panickar J, Lakhanpaul M, Lambert PC, et al. Oral prednisolone for preschool children with acute virus-induced wheezing. N Engl J Med 2009; 360: 329-338.

3 Gadomski AM, Scribani MB. Bronchodilators for bronchiolitis. Cochrane Database Syst Rev 2014; 6: CD001266.

4 Scarfone RJ, Loiselle JM, Joffe MD, et al. A randomized trial of magnesium in the emergency department treatment of children with asthma. Ann Emerg Med 2000; 36: 572-578.

5 Ciarallo L, Brousseau D, Reinert S. Higher-dose intravenous magnesium therapy for children with moderate to severe acute asthma. Arch Pediatr Adolesc Med 2000; 154: 979-983.

6 Gurkan F, Haspolat $\mathrm{K}$, Bosnak M, et al. Intravenous magnesium sulphate in the management of moderate to severe acute asthmatic children nonresponding to conventional therapy. Eur J Emerg Med 1999; 6: 201-205.

7 Devi PR, Kumar L, Singhi SC, et al. Intravenous magnesium sulfate in acute severe asthma not responding to conventional therapy. Indian Pediatr 1997; 34: 389-397.

8 Ciarallo L, Sauer AH, Shannon MW. Intravenous magnesium therapy for moderate to severe pediatric asthma: Results of a randomized, placebo-controlled trial. J Pediatr 1996; 129: 809-814.

9 Griffiths B, Kew KM. Intravenous magnesium sulfate for treating children with acute asthma in the emergency department. Cochrane Database Syst Rev 2016; 4: CD011050.

10 Singhi S, Grover S, Bansal A, et al. Randomised comparison of intravenous magnesium sulphate, terbutaline and aminophylline for children with acute severe asthma. Acta Paediatr 2014; 103: 1301-1306.

11 Torres S, Sticco N, Bosch JJ, et al. Effectiveness of magnesium sulfate as initial treatment of acute severe asthma in children, conducted in a tertiary-level university hospital: A randomized, controlled trial. Arch Argent Pediatr 2012; 110: 291-296.

12 Reinhart RA. Magnesium metabolism. A review with special reference to the relationship between intracellular content and serum levels. Arch Intern Med 1988; 148: 2415-2420. 
13 Lowell DI, Lister G, Von Koss H, et al. Wheezing in infants: The response to epinephrine. Pediatrics 1987; 79: 939-945.

14 Schuh S, Coates AL, Dick P, et al. A single versus multiple doses of dexamethasone in infants wheezing for the first time. Pediatr Pulmonol 2008; 43: 844-850.

15 Delgado A, Chou KJ, Silver EJ, et al. Nebulizers vs metered-dose inhalers with spacers for bronchodilator therapy to treat wheezing in children aged 2 to 24 months in a pediatric emergency department. Arch Pediatr Adolesc Med 2003; 157: 76-80.

16 StatsDirect ltd. StatsDirect Statistical Software. www.statsdirect.com. Altrincham, StatsDirect Ltd, 2013.

17 Albuali WH. The use of intravenous and inhaled magnesium sulphate in management of children with bronchial asthma. J Matern Fetal Neonatal Med 2014; 27: 1809-1815.

18 Lukkarinen M, Koistinen A, Turunen R, et al. Rhinovirus-induced first wheezing episode predicts atopic but not nonatopic asthma at school age. J Allergy Clin Immunol 2017; 140: 988-995.

$19 \mathrm{Su} \mathrm{Z}$, Li R, Gai Z. Intravenous and nebulized magnesium sulfate for treating acute asthma in children: A systematic review and meta-analysis. Pediatr Emerg Care 2016; in press [https://doi.org/10.1097/PEC. 0000000000000909].

20 Mohammed S, Goodacre S. Intravenous and nebulised magnesium sulphate for acute asthma: Systematic review and meta-analysis. Emerg Med J 2007; 24: 823-830.

21 Shan Z, Rong Y, Yang W, et al. Intravenous and nebulized magnesium sulfate for treating acute asthma in adults and children: A systematic review and meta-analysis. Respir Med 2013; 107: 321-330.

22 Saglani S, Malmstrom K, Pelkonen AS, et al. Airway remodeling and inflammation in symptomatic infants with reversible airflow obstruction. Am J Respir Crit Care Med 2005; 171: 722-727.

23 Brand PL, Baraldi E, Bisgaard H, et al. Definition, assessment and treatment of wheezing disorders in preschool children: An evidence-based approach. Eur Respir J 2008; 32: 1096-1110.

24 Destino L, Weisgerber MC, Soung P, et al. Validity of respiratory scores in bronchiolitis. Hosp Pediatr 2012; 2 : 202-209.

25 Cunningham S, Nair H, Campbell H. Deciphering clinical phenotypes in acute viral lower respiratory tract infection: Bronchiolitis is not an island. Thorax 2016; 71: 679-680.

26 Alansari K, Sayyed R, Davidson BL, et al. Intravenous magnesium sulfate for bronchiolitis: a randomized trial. Chest 2017; 152: 113-119.

27 Castro-Rodriguez JA. The asthma predictive index: A very useful tool for predicting asthma in young children J Allergy Clin Immunol 2010; 126: 212-216.

28 Panico L, Stuart B, Bartley M, et al. Asthma trajectories in early childhood: identifying modifiable factors. PLoS One 2014; 9: e111922.

29 Nicolai A, Frassanito A, Nenna R, et al. Risk factors for virus-induced acute respiratory tract infections in children younger than 3 years and recurrent wheezing at 36 months follow-up after discharge. Pediatr Infect Dis J 2017; 36 : 179-183.

30 Jääskeläinen MVS. Tobacco Statistics 2015. Helsinki, Official Statistics of Finland, National Institute for Health and Welfare, 2015.

31 Eddleston J, Lee RU, Doerner AM, et al. Cigarette smoke decreases innate responses of epithelial cells to rhinovirus infection. Am J Respir Cell Mol Biol 2011; 44: 118-126.

32 Rower JE, Liu X, Yu T, et al. Clinical pharmacokinetics of magnesium sulfate in the treatment of children with severe acute asthma. Eur J Clin Pharmacol 2017; 73: 325-331. 